\title{
Multi-wavelength follow-up studies of eruptive cataclysmic variables in the MeerKAT and CTA era
}

\section{H. Szegedi*}

University of the Free State, Bloemfontein, South Africa

E-mail: SzegediH@ufs.ac.za

\section{A. Odendaal}

University of the Free State, Bloemfontein, South Africa

E-mail: WinkA@ufs.ac.za

\section{P.J. Meintjes}

University of the Free State, Bloemfontein, South Africa

E-mail: MeintjpJ@ufs.ac.za

Cataclysmic variables (CVs) have been studied for decades, but it is only during recent years that the importance of multi-wavelength studies of these sources have motivated dedicated surveys e.g. CRTS and MASTER. Multi-wavelength follow-up studies are required to fully constrain the properties and radiative processes of $\mathrm{CVs}$, as these sources emit energy over almost the whole electromagnetic spectrum. A selection of results from a multi-wavelength follow-up study of $\mathrm{CV}$ systems, showing high levels of transient emission, will be discussed. This will also be used to demonstrate how essential dedicated radio and gamma-ray telescopes like MeerKAT and the forth-coming Cherenkov Telescope Array (CTA) are to complete our understanding of these sources and associated eruptive processes in general. Constraining non-thermal synchrotron emission processes in CVs and high energy outbursts are of special interest.

5th Annual Conference on High Energy Astrophysics in Southern Africa

4-6 October 2017

University of the Witwatersrand (Wits), South Africa

\footnotetext{
* Speaker.
} 


\section{Introduction}

Cataclysmic variables (CVs) are the only accreting systems in the Universe that only recently lifted their veils of secrecy at the shortest and longest wavelengths. Radio observations of nonmagnetic CVs (NMCVs) in the 1980s had low detection rates and only three NMCVs were detected (SU UMa, EM Cyg and TY Psc) at radio wavelengths ([1], [2] and [3]). No detections were made during follow-up observations of the same sources, at higher sensitivity levels ([4] and references therein). Almost two decades after the initial radio observations, NMCVs observed during outburst were detected at radio and gamma-ray wavebands ([5], [4], [6] and [7]) and sparked a renewed interest in the mechanisms that drive non-thermal emission. NMCVs are the focus for this discussion as many of these sources show high levels of transient emission that could be attributed to magnetohydrodynamical (MHD) processes.

CVs are compact binary systems that consist of a white dwarf (WD) accreting material from a low-mass main-sequence star, due to Roche-lobe overflow. The WD primary has a mass range of $\sim 0.8 \mathrm{M}_{\odot}$ to $\sim 1.2 \mathrm{M}_{\odot}$ ([8] and [9]) and the secondary is usually a typical $\mathrm{K}$ - or M spectral type star with a mass $\leq 1 M_{\odot}$ ([10] and [11]). The separation between the stellar components ranges from $\sim 1 \mathrm{R}_{\odot}$ to $1 \mathrm{AU}$, and the orbital period ranges from $\sim 80 \mathrm{~min}$ to $\sim 2.6$ days [12]. The magnetic field strength of the WD dictates whether an accretion disc is formed, and if so, also the proximity of the disc's inner edge to the surface of the WD. WDs in NMCVs have weak magnetic fields $(B<1 \mathrm{MG})$ that allow accretion discs to be formed in these systems. NMCVs are subdivided into four classes, namely classical novae $(\mathrm{CNe})$, recurrent novae (RNe), dwarf novae (DNe), and nova-like variables (e.g. [13], p. 27). Magnetic CVs have WDs with strong magnetic fields and are subdivided into intermediate polars (IPs) $(B \sim 1-10 \mathrm{MG})$ or polars $(B \sim 10-240 \mathrm{MG})$ (e.g. [13], p. 28 and [14]). Most IPs accrete material onto the WD's polar caps via a truncated accretion disc and an accretion curtain. No accretion disc is formed in polars and material is carried by an accretion stream directly onto the primary's polar caps. Only $20 \%-25 \%$ of the CV population is magnetic, therefore in most observed CVs an accretion disc is detected [15].

The outbursts in NMCVs are essentially caused by either runaway thermonuclear burning of the accreted material on the WD's surface ( $\mathrm{CNe}$ and $\mathrm{RNe}$ ), or a release of gravitational energy caused by a temporary increase in the mass transfer rate of the accretion disc (DNe). The sudden increase in the mass transfer rate in DNe is caused by an instability in the accretion disc due to a build-up of material in the disc, leading to an increase in viscosity which enables an increase in the rate of outward angular-momentum transport (e.g. [16], p.55). Nova outbursts do not occur as frequently as DN outbursts, but undergo an average brightening of 10-12 magnitudes (e.g. [17], p. 744), which is much brighter than a DN outburst (2-8 magnitudes) [6]. During an outburst of a $\mathrm{CN}$ or $\mathrm{RN}$, but not a $\mathrm{DN}$, the outflow of material can interact violently with the interstellar medium and have been detected by Fermi-LAT [7]. The gamma-ray emission is most likely caused by high-energy protons in ejecta interacting with nuclei, resulting in the formation of neutral pions. The gamma-rays that are produced by this hadronic process is an indicator of strong shocks which accelerate particles to relativistic energies ([18] and references therein). Recent radio (VLA) detections of compact, high brightness temperature knots from V959 Mon, by Chomiuk et al. [19], confirmed the presence of non-thermal shock emission in a gamma-ray producing nova.

In accreting systems, the mass transfer rate is a key factor that determines the low, faint (qui- 
escence) and high, bright (outburst) states. In a low state, the mass transfer rate can be as low as $\sim 10^{-11} \mathrm{M}_{\odot} \mathrm{yr}^{-1}$, and during a high state the mass transfer rate can reach $\sim 10^{-8} \mathrm{M}_{\odot} \mathrm{yr}^{-1}$ ([20] and [21]). "Non-magnetic" does not mean "no magnetic field" and therefore it is expected that the magnetic field will have an influence on the ionized particles in the accretion disc, influencing plasma dynamics driving particle acceleration. We have a fairly good grasp on the origin and mechanisms that are responsible for radiation from infrared to X-ray (see [13] and [22] for a review), but the production processes of non-thermal emission, especially in CVs during outburst, have still to be investigated.

In the following section, MHD turbulence as a mechanism for outward angular momentum transport in the disc, as well as magneto-gravitational instabilities leading to DNe outbursts and the associated production processes of radio and gamma-ray emission in NMCVs, will be discussed. In Section 3 some optical results of a current multi-wavelength follow-up study of NMCVs will be used to demonstrate how optical surveys can be utilized to identify possible radio and gamma-ray emitting NMCVs.

\section{Turbulent magnetized accretion and its role in DNe outbursts}

\subsection{Turbulent accretion discs and DNe outbursts}

The accretion discs in some NMCVs switch between a low, faint state and a bright, hot state driven by a thermal-viscous instability [23]. When a DN is in quiescence the disc is in a cold, low-viscosity state. The material cannot interact effectively with the magnetic field as the electrons combine with nuclei to create electrically neutral atoms. If the material is heated to $\geq 7000 \mathrm{~K}$, hydrogen gas becomes ionized and the disc's opacity to radiation starts to increase (e.g. [16], p. 63). An increase in temperature will also increase viscosity and the opacity traps the heat created by viscous interactions. This will cause an even further increase in temperature which will bring the disc from partially ionized to fully ionized. The higher viscosity of the ionized disc will drive the flow of material inward at a higher rate. The disc is then in a hot, highly viscous state and the DN is in outburst. This state cannot be sustained for long as the inward flow rate of material exceeds the mass transfer rate through the L1 point and in effect will cause the disc to return to its original quiescent state.

Viscosity is a crucial factor in mass accretion [24], but molecular viscosity alone is not adequate to sustain the inferred accretion rates in these systems that would result in an outburst. Turbulent viscosity does provide a mechanism that enables effective angular momentum transport from the inner parts of the disc to the outer parts, causing the disc material to spiral faster inward towards the WD. Keplerian discs are stable against hydrodynamic instabilities, i.e. any perturbance like a density varied stream of material, will be damped out by the Coriolis effect on a dynamical timescale [25]. Balbus and Hawley [26] demonstrated that turbulence driven by magnetohydrodynamic (MHD) instabilities, on the other hand, provides a sustainable mechanism to transport angular momentum.

The transport of angular momentum through the Balbus and Hawley model occurs via a socalled magnetorotational instability (MRI), which manifests itself through complicated computational simulations. A more simplistic analytical model [27] explained the MRI in terms of a 
magneto-gravitational instability, which will be explained briefly hereafter. When the accretion disc is ionized, it behaves like a plasma. If the magnetic Hartmann number, i.e. the ratio of magnetic stresses to particle viscosity in a magnetized field, is $M \geq 1$, the magnetic field starts to influence the dynamics of the plasma [28]. The magnetic field lines are "frozen" into the accretion disc and due to the Keplerian flow of material the field lines are dragged along in the disc ([29] and [30]), and amplified until it becomes unstable to magnetic reconnection. This will result in particle acceleration and non-thermal synchrotron emission under certain conditions. For a full analytical treatment and summary, see [28].

The transient emission of DNe can be evaluated in terms of a magneto-gravitational instability. Meintjes and Breedt [31] have shown that the accretion discs in slow rotators may cycle between states of low magnetization $\left(M_{\text {cold }} \ll 1\right)$ and high magnetization $\left(M_{\text {hot }} \geq 1\right)$ when the disc cycles through low and high viscosity states. These authors showed that during low magnetization the disc is in a low viscosity state and the frozen-in magnetic field can readily be advected along with the accretion flow, resulting in an effective magnetic field transport mechanism in the disc. Inside the corotation radius around the WD, the azimuthal disc flow velocity is faster than the rotation velocity of the WD, and slower outside the corotation radius. The magnetic field will exert an outward magneto-centrifugal push on the inward flowing viscosity-driven material. The material rams into the magnetic obstruction, causing a build-up of disc material and an increase in turbulence as the material no longer adheres to the Keplerian flow outside the corotation radius. The azimuthal flow across the frozen-in disc field is decelerated, causing the effective gravity to point radially inward. The effective gravity can be quantified by

$$
\mathbf{g}_{\mathrm{eff}, \perp}=\left(-\frac{G M_{1}}{R^{2}}+\frac{v_{\phi}^{2}}{R}\right) \hat{\mathbf{e}}_{\mathrm{R}},
$$

where $M_{1}$ is the mass of the compact object and $R$ the distance from the center of the compact object in the equatorial plane of the binary. From the equation, one can see that for a Keplerian flow (i.e. $\mathrm{v}_{\phi} \rightarrow \mathrm{v}_{\mathrm{K}}$ ), $\mathbf{g}_{\mathrm{eff}, \perp} \rightarrow 0$, but when the turbulent material no longer adheres to the Keplerian flow outside the corotation radius (i.e. $\mathrm{v}_{\phi}<\mathrm{v}_{\mathrm{K}}$ ), then $\mathbf{g}_{\mathrm{eff}, \perp}<0$ and the plasma will experience a net inward pull towards the WD. This large scale inward advection will drain the bulk of the disc plasma and also causes the magnetic field anchored in the disc to be advected with the inward flow, leading to the formation of magnetic bubbles and subsequently reconnection.

Magnetic turbulence can also lead to the formation of magnetic islands, or eddies. The swirling motion of turbulent eddies in the accretion disc causes a flickering in the lightcurves of DNe, which display a characteristic red-noise behaviour defined by the Kolmogorov $E(k) \propto k^{-5 / 3}$ law (e.g. [31] and references therein). The eddies can also become buoyant due to the Parker buoyancy instability [32]. Vertical magnetic field components will be created producing a magnetized corona above and below the accretion disc [33].

A fast-rotating compact object will not allow decelerated material to trickle past the magnetic centrifugal barrier during a low state [34]. The inner disc will be truncated just outside the corotation radius causing a build-up of material (Figure 1). At some point the inward pressure of the disc will overcome the magnetic barrier, pulling all the built-up material inward towards the compact object. This sudden increase in the accretion rate will manifest as an outburst or a flare. When the inward flowing material is depleted, the barrier will recover at the corotation radius. This cycle is 


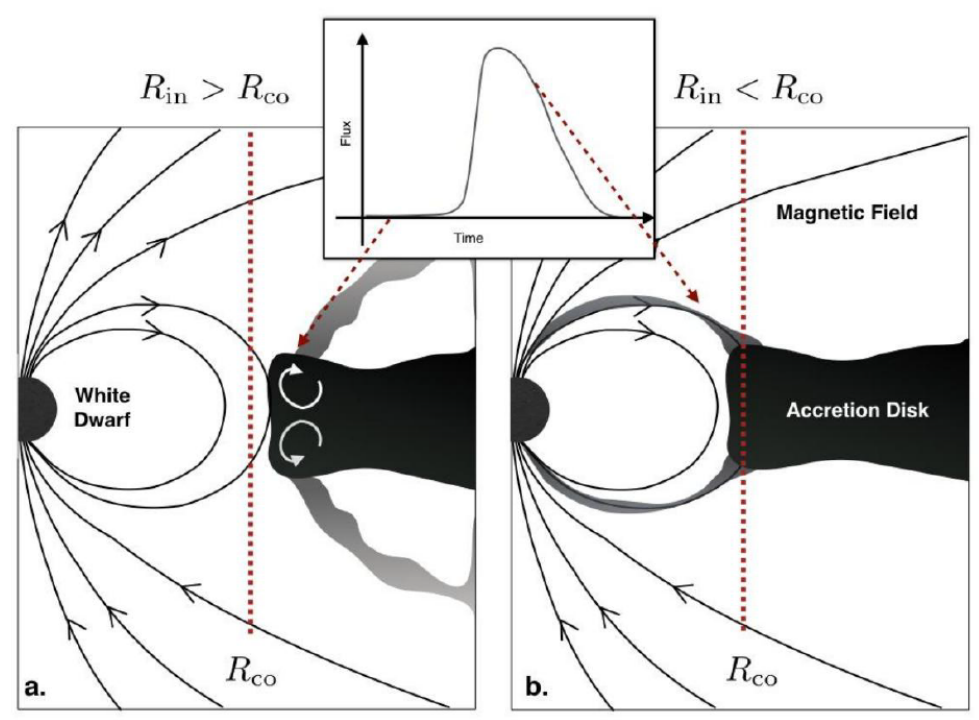

Figure 1: An illustration of magnetically gated accretion in a non-magnetic CV. a.) A magnetic centrifugal barrier is created at the corotation radius that pushes against the inflowing viscosity-driven material, causing a build-up of material. b.) At some point, the inward pushing gas will overthrow the magnetic barrier and allow a burst of accretion onto the WD surface. When the inward flowing material is depleted the barrier will recover at the corotation radius. This cycle is repeated on a time-scale comparable to the viscosity-driven accretion cycle. (Adopted from [34], Fig. 3).

repeated on a time-scale comparable to the viscosity-driven accretion cycle. This phenomenon has been suggested in a nova-like NMCV, MV Lyrae, by Scaringi et al. [34]. MV Lyrae displayed a 2 magnitude increase in brightness every 2 hours that lasted for $\sim 30$ minutes.

\subsection{Turbulence-driven non-thermal outbursts}

As mentioned before, the inward motion during the highly viscous state of the disc will cause the magnetospheric fields anchored in the disc to become highly distorted. This will lead to centrifugally accelerated outward winds from the disc ([31] and references therein). Meintjes [28] utilized the Van der Laan model to show that the initial flux of an expanding synchrotron bubble, associated with a source at a distance of $\sim 100 \mathrm{pc}$, is approximately

$S_{\mathrm{m}, \mathrm{o}} \approx 60\left[\left(\frac{\varepsilon_{\mathrm{e}}}{1 \mathrm{MeV}}\right)^{1.75}\left(\frac{r_{\mathrm{o}}}{10^{9} \mathrm{~cm}}\right)^{6.67}\left(\frac{N_{\mathrm{o}}}{10^{9} \mathrm{~cm}^{-3}}\right)\left(\frac{B_{\mathrm{o}}}{100 \mathrm{Gauss}}\right)^{0.958}\right]^{0.3529} \times\left(\frac{v_{\mathrm{mo}}}{60 \mathrm{GHz}}\right)^{1.3088} \mu \mathrm{Jy}$,

for an electron power-law spectrum with index $\delta=2.75$. The frequency where the initial flux peaks is then

$$
v_{\mathrm{mo}} \approx 60\left(\frac{\varepsilon_{\mathrm{e}}}{\mathrm{MeV}}\right)^{0.5185}\left(\frac{N_{\mathrm{o}}}{10^{9} \mathrm{~cm}^{-3}}\right)^{0.2963}\left(\frac{r_{\mathrm{o}}}{10^{9} \mathrm{~cm}}\right)^{0.2963}\left(\frac{B_{\mathrm{o}}}{100 \mathrm{G}}\right)^{0.703} \mathrm{GHz}
$$

where $\varepsilon_{\mathrm{e}}$ is the electron energy, $r_{\mathrm{o}}$ is the initial radius of the bubble, $N_{\mathrm{o}}$ is the electron density and $B_{\mathrm{o}}$ is the initial magnetic field. 

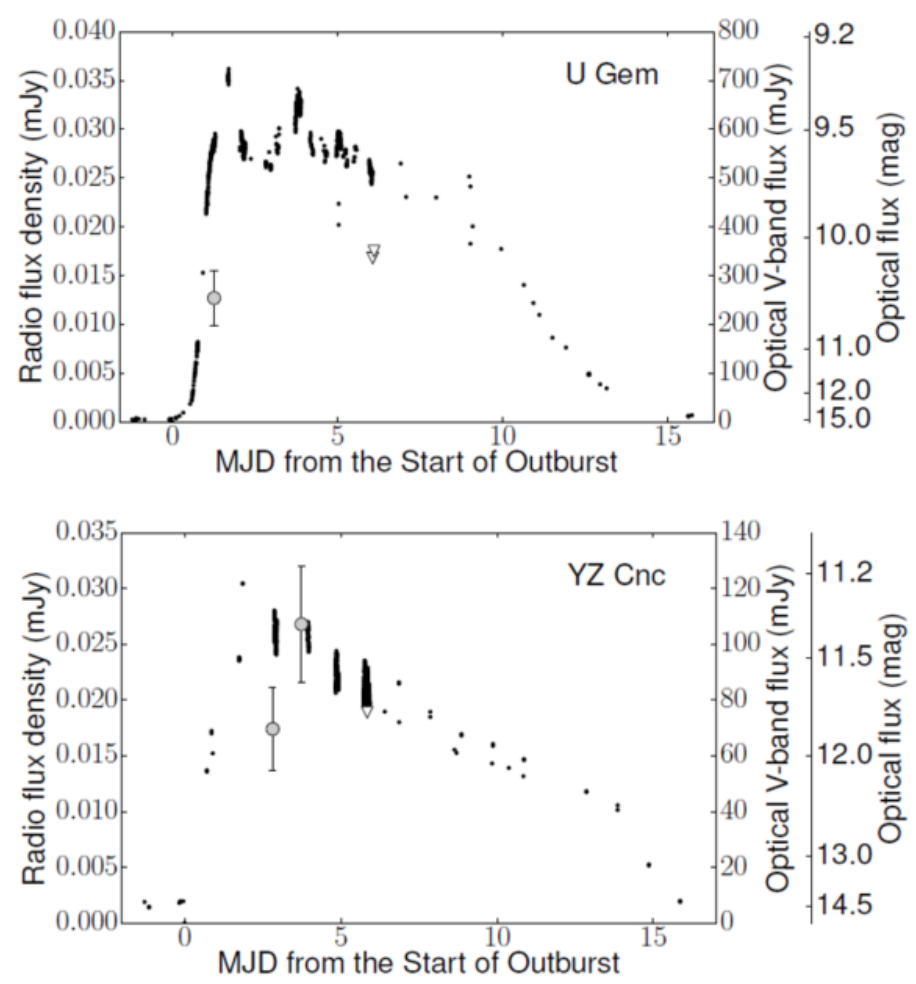

Figure 2: The optical ( $V$-band, black dots) and radio (10 GHz, grey circles) lightcurves of U Gem (outburst) and YZ Cnc (superoutburst). (Adapted from [6], Fig. 1).

The centrifugally driven disc winds generated during DN eruptions will therefore be observable with sensitive radio telescopes, e.g. MeerKAT and the SKA, that will have sensitivity levels of $\sim 100 \mu \mathrm{Jy}$ ( 1 day) and $\sim 1 \mu \mathrm{Jy}$ ( 1 day), respectively [35]. Coppejans et al. [6] observed outflows for five DNe in outburst with the VLA. The optical and radio lightcurves of two sources, $\mathrm{U}$ Gem and YZ Cnc, are shown in Figure 2. These detections, together with the radio detections of novalike systems [4], have proven that NMCVs are radio emitters and that the detected emission is consistent with synchrotron emission. The Fermi-LAT detections of gamma-ray emission at energies above $100 \mathrm{MeV}$ from novae, also opens up the possibilities to study and constrain particle acceleration in non-relativistic shocks that form in nova outbursts.

\section{Optical follow-up observations}

We are on the brink of a new generation of radio (e.g. MeerKAT, SKA) and gamma-ray telescopes (e.g. CTA) coming online, and to launch successful multi-wavelength campaigns it is important to point out how the results of optical observations can be used to identify possible radio and gamma-ray emitting NMCVs. For our current multi-wavelength follow-up study of NMCVs, we utilized the Catalina Real-Time Transient Survey (CRTS) to identify CV candidates that showed high levels of transient emission. The selection criteria included sources that were observed for more than a year by CRTS, exhibited variability of more than 2 magnitudes, undergoing regular outbursts, and were observable from South Africa. A total of $18 \mathrm{CVs}$ were observed, but for the 


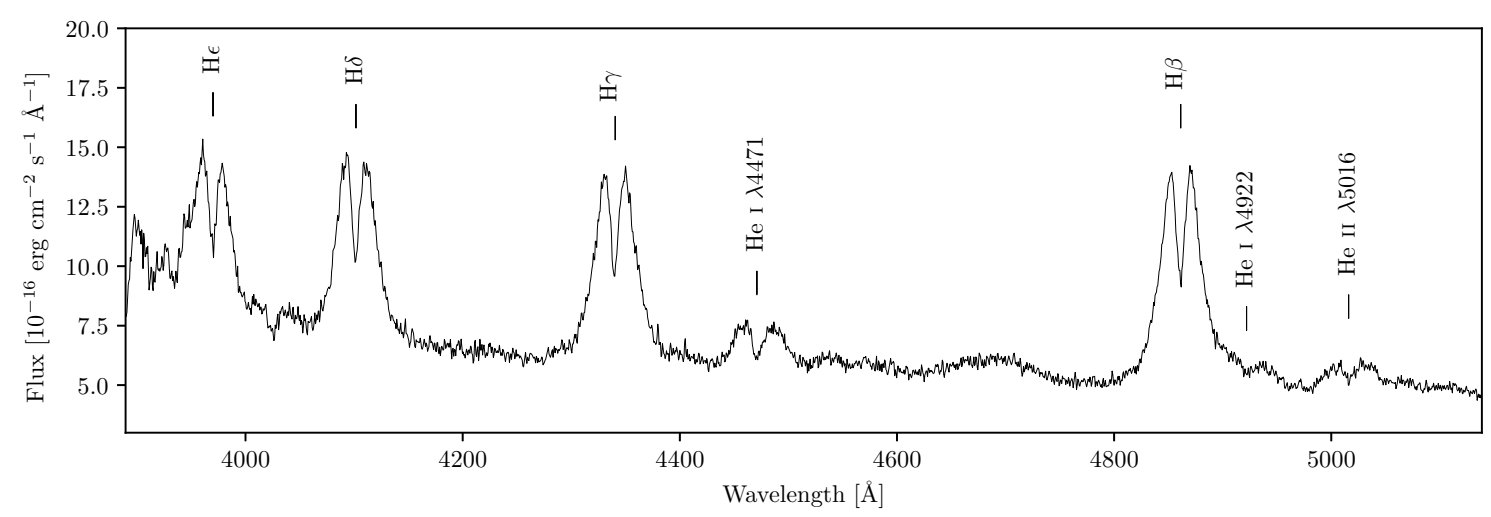

Figure 3: The high-resolution spectrum of AR Pic, taken during quiescence on 26 February 2017. The double-peak profile of the emission lines are caused by the Keplerian flow of the accreton disc.

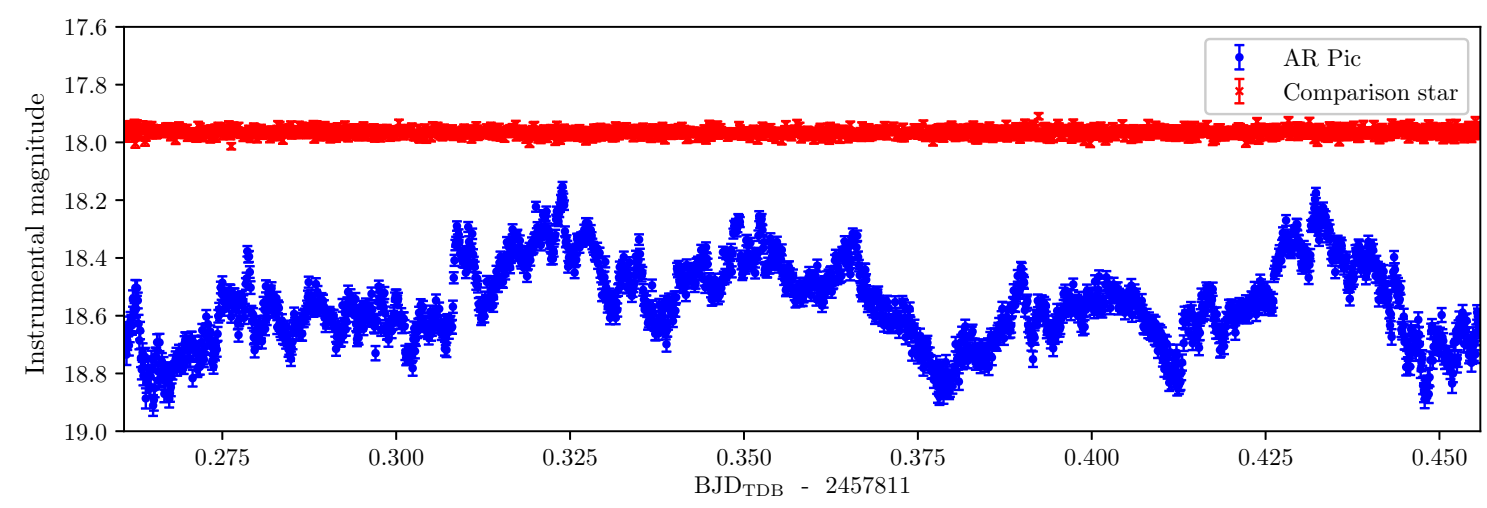

Figure 4: The lightcurve of AR Pic, taken during quiescence on 26 February 2017. The flickering is evidence of a turbulent accretion disc.

purpose of this discussion, the optical results of only two SU UMa DNe, AR Pic and QW Ser, will be used as demonstration.

AR Pic and QW Ser were observed with the 1.0-m telescope and SHOC (photometry), and the 1.9-m telescope and SpUpNIC (spectroscopy), at the South African Astronomical Observatory (SAAO), Sutherland. Both sources showed interesting transient behaviour during observations. The double-peak profiles of the emission lines in the spectrum of AR Pic (Figure 3) shows a well formed accretion disc. The double-peak profiles are due to the Keplerian flow in the disc and indicate that the disc material reaches velocities of $\sim 2000 \mathrm{~km} \mathrm{~s}^{-1}$. The flickering in the lightcurve (Figure 4), taken during quiescence, is evidence of the eddies in a turbulent disc (e.g. [16], p. 151, and [36], p. 164) that will contribute to the red noise character of the lightcurves [27].

During spectroscopic observations on August 8, 2016, QW Ser went into outburst. It experienced a brightness increase of $\sim 5$ magnitudes and returned to quiescence within a week. Simultaneous photometric and spectroscopic observations were performed every night until it returned to quiescence. The deep absorption lines of the Balmer series in the spectrum (Figure 5) are typical of a DN outburst. This indicates that during a high state the region around the WD expands, effectively creating a hot central region surrounded by a cooler gas. The absorption features are therefore 


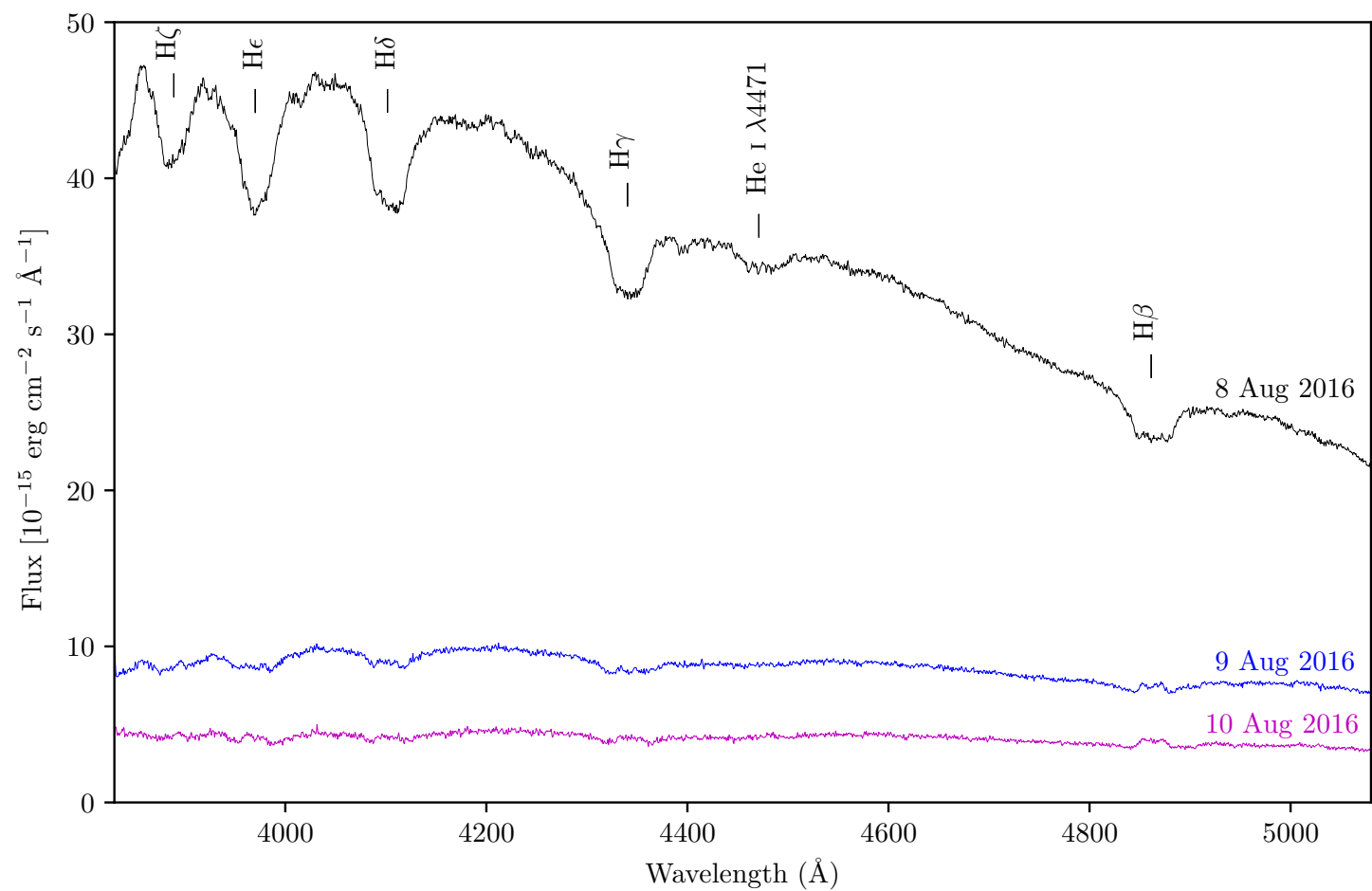

Figure 5: The high-resolution spectra of QW Ser in outburst (8 Aug 2016) and in the process of settling back to quiescence ( 9 and 10 Aug 2016). The deep absorption features indicate that the disc is optically thick during outburst. After outburst the disc cooled down and became optically thin. The absorption features turned to double-peak emission lines, evidence of an accretion disc.

formed when the cooler gas absorbs emission from the optically thick inner (hot) region. The expanded region can be attributed to the magnetic bubbles that drifted vertically and the centrifugally accelerated outward winds. During the outburst, the outflows reach velocities of $\sim 1000 \mathrm{~km} \mathrm{~s}^{-1}$. Although not detected, it is probable that shocks formed in the outflows which may possibly result in detectable gamma-ray emission. As the disc cooled, it became optically thin and the emission lines became visible. The double-peak profile of the $\mathrm{H} \beta$ emission line is yet again evidence of the accretion disc in the system.

\section{Conclusion}

The recent radio and gamma-ray detections in NMCVs spurred a renewed interest in these accreting systems. It has been shown that even a weak magnetic field of the compact object in NMCVs, will have an influence on the accreting material in the disc. It will contribute to the turbulent viscosity and the created turbulence in the accretion discs will eventually lead to outbursts. The synchrotron emission from the magnetized blobs of material, as well as the outflows interacting with the surrounding interstellar material may be detectable in the new era of sophisticated radio and gamma-ray telescopes, e.g. MeerKAT, later SKA, and CTA.

The transient behaviour visible in the optical results, and the characteristics of the system derived from the results, can be used to identify radio and gamma-ray emitting CVs that can be ob- 
served with MeerKAT, more specifically ThunderKAT (The hunt for dynamic and explosive radio transients with MeerKAT [37]), and CTA. This approach will be imperative to identify candidates through the enormous amount of data acquired by optical surveys like the forth-coming Large Synoptic Survey Telescope (LSST).

\section{Acknowledgements}

The first author expresses her appreciation towards the DST/NRF for funding through SAGAMMA. This paper uses observations made at the South African Astronomical Observatory (SAAO), Sutherland.

\section{References}

[1] A. O. Benz, E. Fuerst and A. L. Kiplinger, First detection of radio emission from a dwarf nova, Nature 302 (1983) 45.

[2] K. C. Turner, $12 \mathrm{~cm}$ Observations of Stellar Radio Sources, in Radio Stars (R. M. Hjellming and D. M. Gibson, eds.), vol. 116 of Astrophysics and Space Science Library, p. 283, 1985, DOI.

[3] A. O. Benz and M. Guedel, VLA detection of radio emission from a dwarf nova, A\&A 218 (1989) $137-140$.

[4] D. L. Coppejans, E. G. Körding, J. C. A. Miller-Jones, M. P. Rupen, C. Knigge, G. R. Sivakoff et al., Novalike cataclysmic variables are significant radio emitters, MNRAS 451 (2015) 3801-3813, [1506.00003].

[5] E. Körding, M. Rupen, C. Knigge, R. Fender, V. Dhawan, M. Templeton et al., A Transient Radio Jet in an Erupting Dwarf Nova, Science 320 (2008) 1318, [0 806.1002 ].

[6] D. L. Coppejans, E. G. Körding, C. Knigge, M. L. Pretorius, P. A. Woudt, P. J. Groot et al., Statistical properties of dwarf novae-type cataclysmic variables: the outburst catalogue, MNRAS 456 (2016) 4441-4454.

[7] M. Ackermann, M. Ajello, A. Albert, L. Baldini, J. Ballet, G. Barbiellini et al., Fermi establishes classical novae as a distinct class of gamma-ray sources, Science 345 (2014) 554-558, [1408.0735].

[8] C. Knigge, The Evolution of Cataclysmic Variables, in Evolution of Compact Binaries (L. Schmidtobreick, M. R. Schreiber and C. Tappert, eds.), vol. 447 of Astronomical Society of the Pacific Conference Series, p. 3, 2011, 1108.4716.

[9] M. Zorotovic, M. R. Schreiber and B. T. Gänsicke, Post common envelope binaries from SDSS. XI. The white dwarf mass distributions of CVs and pre-CVs, A\&A 536 (2011) A42, [1108.460 0].

[10] F. Giovannelli and L. Sabau-Graziati, The Golden Age of Cataclysmic Variables and Related Objects: The State of Art., in The Golden Age of Cataclysmic Variables and Related Objects - III (Golden2015), p. 1, 2015.

[11] J. Smak, Outbursts of dwarf novae, PASP 96 (1984) 5-18.

[12] H. Ritter and U. Kolb, VizieR Online Data Catalog: Cataclysmic Binaries, LMXBs, and related objects (Ritter+, 2004), VizieR Online Data Catalog 1 (2011).

[13] B. Warner, Cataclysmic Variable Stars, vol. 28. Cambridge University Press, Cambridge, 1995. 
[14] B. T. Gänsicke, G. D. Schmidt, S. Jordan and P. Szkody, Phase-resolved Hubble Space Telescope/STIS Spectroscopy of the Exposed White Dwarf in the High-Field Polar AR Ursae Majoris, ApJ 555 (2001) 380-392, [astro-ph/0103131].

[15] L. Ferrario, D. de Martino and B. T. Gänsicke, Magnetic White Dwarfs, Space Science Reviews 191 (2015) 111-169, [1504.08072].

[16] C. Hellier, Cataclysmic Variable Stars - How and why they vary. Praxis Publishing Ltd, Chichester, 2001.

[17] B. W. Carroll and D. A. Ostlie, An introduction to modern astrophysics and cosmology. 2006.

[18] B. D. Metzger, T. Finzell, I. Vurm, R. Hascoët, A. M. Beloborodov and L. Chomiuk, Gamma-ray novae as probes of relativistic particle acceleration at non-relativistic shocks, MNRAS 450 (2015) 2739-2748, [1501.05308].

[19] L. Chomiuk, J. D. Linford, J. Yang, T. J. O’Brien, Z. Paragi, A. J. Mioduszewski et al., Binary orbits as the driver of $\gamma$-ray emission and mass ejection in classical novae, Nature 514 (2014) 339-342, [1410.3473].

[20] B. T. Gänsicke, E. M. Sion, K. Beuermann, D. Fabian, F. H. Cheng and J. Krautter, TT Arietis: the low state revisited, $A \& A 347$ (1999) 178-184.

[21] J. R. Thorstensen, F. A. Ringwald, R. A. Wade, G. D. Schmidt and J. E. Norsworthy, PG0027 + $260-$ an example of a class of cataclysmic binaries with mysterious, but consistent, behavior, Astronomical Journal 102 (1991) 272-283.

[22] P. Szkody and B. T. Gänsicke, Cataclysmic Variables, Journal of the American Association of Variable Star Observers (JAAVSO) 40 (2012) 563-571.

[23] J. Smak, Accretion in cataclysmic binaries. IV - Accretion disks in dwarf novae, AcA 34 (1984) 161-189.

[24] W. H. G. Lewin, J. van Paradijs and E. P. J. van den Heuvel, X-ray binaries., Cambridge Astrophysics Series 26 (1995).

[25] S. A. Balbus, J. F. Hawley and J. M. Stone, Nonlinear Stability, Hydrodynamical Turbulence, and Transport in Disks, ApJ 467 (1996) 76.

[26] S. A. Balbus and J. F. Hawley, Instability, Turbulence, and Enhanced Transport in Accretion Disks, in IAU Colloq. 163: Accretion Phenomena and Related Outflows (D. T. Wickramasinghe, G. V. Bicknell and L. Ferrario, eds.), vol. 121 of Astronomical Society of the Pacific Conference Series, p. 90, 1997.

[27] P. J. Meintjes, E. Breedt and M. Erwee, Magnetized Accretion Discs and the Dwarf Nova Phenomenon, in Proceedings of Frontier Research in Astrophysics (FRAPWS2014) held 26-31 May, 2014 in Mondello (Palermo), Italy, p. 10, 2014.

[28] P. J. Meintjes, Transient Sources in Astrophysics: From Radio to Gamma-Rays, in 3rd Annual Conference on High Energy Astrophysics in Southern Africa (HEASA2015), p. 4, 2015.

[29] E. Priest and T. Forbes, eds., Magnetic reconnection : MHD theory and applications, 2000.

[30] A. Odendaal and P. Meintjes, Cataclysmic variables: New frontiers in multi-wavelength research, in Proceedings of the 4th Annual Conference on High Energy Astrophysics in Southern Africa (HEASA 2016). January 13th, 2016. South African Astronomical Observatory (SAAO), Cape Town, South Africa., p. 30, 2016. 
[31] P. J. Meintjes and E. Breedt, Magnetic viscosity: outbursts and outflows in accretion driven systems, Mem.S.A.It. 86 (2015) 89.

[32] E. N. Parker, The Dynamical State of the Interstellar Gas and Field, ApJ 145 (1966) 811.

[33] A. A. Galeev, R. Rosner and G. S. Vaiana, Structured coronae of accretion disks, ApJ 229 (1979) 318-326.

[34] S. Scaringi, T. J. Maccarone, C. D’Angelo, C. Knigge and P. J. Groot, Magnetically gated accretion in an accreting 'non-magnetic' white dwarf, Nature 552 (2017) 210-213, [1712 . 04949 ].

[35] R. Fender, The Scientific Potential of LOFAR for Time Domain Astronomy, in New Horizons in Time Domain Astronomy (E. Griffin, R. Hanisch and R. Seaman, eds.), vol. 285 of IAU Symposium, pp. 11-16, 2012, 1112.2580, DOI.

[36] A. R. Choudhuri, The physics of fluids and plasmas : an introduction for astrophysicists. 1998.

[37] R. Fender, P. A. Woudt, R. Armstrong, P. Groot, V. McBride, J. Miller-Jones et al., ThunderKAT: The MeerKAT Large Survey Project for Image-Plane Radio Transients, ArXiv e-prints (2017), [1711.04132]. 